\title{
Rupture pressure values of cerebral arteries in the presence of unruptured intracranial aneurysm
}

\section{Leszek Lombarski}

Medical University of Warsaw

Przemysław Kunert ( $\sim$ przemyslaw.kunert@wum.edu.pl )

Medical University of Warsaw

\section{Sylwia Tarka}

Medical University of Warsaw

\section{Adam Piechna}

Warsaw University of Technology

\section{Sławomir Kujawski}

Collegium Medicum in Bydgoszcz, Nicolaus Copernicus University in Toruń

\section{Andrzej Marchel}

Medical University of Warsaw

\section{Research Article}

Keywords: cerebral artery, arterial bifurcation, cerebrovascular disease, intracranial aneurysm, rupture pressure

Posted Date: February 15th, 2022

DOI: https://doi.org/10.21203/rs.3.rs-1200563/v1

License: (c) (i) This work is licensed under a Creative Commons Attribution 4.0 International License.

Read Full License 


\section{Abstract}

Cerebral arteries (CAs) are prone to the saccular aneurysm formation. Since aneurysms may be considered as balloon-like dilations of the locally weakened arterial wall, it should be determined whether the presence of intracranial aneurysm is related to the generalized weakening of CAs. Among 184 consecutive forensic autop-sies, eight brains with a single unruptured saccular aneurysm were identified. Aneurysms with adjacent CAs and specific CA segments were excised, namely: the anterior communicating artery, basilar artery, internal carotid arteries, and middle cerebral arteries. Then, aneurysm and CA specimens were subjected to pressure-inflation tests until rupture occurred at the arterial bifurcation or at the wall of the CA or aneurysm. The same protocol was applied to the control group composed of CAs excised from eight brains without aneurysm. No significant differences were noted between the experimental and control groups, depending on the mean rup-ture pressure (1054 vs. $1048 \mathrm{mmHg}$ ) and rupture site (bifurcation vs. wall) of the analyzed specimens. These findings indicate that the presence of unruptured saccular aneurysm is not related to generalized weakening of CAs among autopsy subjects. Moreover, the CA bifurcations do not represent regions of decreased wall strength.

\section{Introduction}

Cerebral arteries (CAs) are particularly prone to aneurysm formation, with an incidence of $3.6-6 \%$ in the general population. ${ }^{1}$ Furthermore, saccular intracranial aneurysm (sIA) rupture is the most prevalent cause of non-traumatic subarachnoid hemorrhage associated with a high mortality rate. ${ }^{2}$ The aforementioned predilection of CAs to sIA development may result from differences in structure-related biomechanical properties of the arteries in diverse vascular beds.

Compared to peripheral arteries, CAs are stiffer due to the lack of elastin, which is mainly confined to the internal elastic lamina (IEL). ${ }^{3}$ Additionally, progressive elastin degradation leads to further stiffness increase of CAs with age. ${ }^{4}$ According to the literature data, CAs of patients harboring sIA may display particular morphological features. ${ }^{5,6}$ However, there is a paucity of data concerning the distinct biomechanical properties of CAs obtained from patients with sIA. ${ }^{7}$ Since mechanisms of the initiation, growth, and rupture of sIAs comprise the interplay between physical and biological processes, ${ }^{8}$ studies concerning the biomechanical properties of CAs may shed light on the uncertain pathogenesis of sIAs. In the present study, we aimed to compare rupture pressure values of specific CA segments harvested from autopsy subjects with and without unruptured SIA and determine whether presence of unruptured SIA is associated with generalized weakening of the wall of CAs.

\section{Materials And Methods}

\section{CA specimens}

During 184 consecutive forensic autopsies, we analyzed brains of patients who died due to extracerebral reasons. Duration between the time of death and biomechanical tests did not exceed 36 hours. All 
cadavers were stored at $4^{\circ} \mathrm{C}$ before the autopsy. Single unruptured sIA was identified in eight brains (age $62 \pm 4$ years; 2 females). The control group consisted of eight brains without sIA, matched in terms of age and sex of the autopsy subjects (age 62 \pm 7 years; 2 females). The sIAs with adjacent CAs were excised using a surgical microscope (Carl Zeiss OPMI pico S100, Germany). Then, specific CA segments characterized by increased risk of sIA formation were collected, including the anterior communicating artery (ACommA) with anterior cerebral arteries, basilar artery (BA) bifurcation, and bifurcation of both internal carotid arteries (ICAs) and middle cerebral arteries (MCAs). A total of 96 specimens were prepared for the tests. Table 1 illustrates the demographic data, including age, sex, cause of death, and aneurysm location, in the experimental group. All experiments were performed in accordance with relevant guidelines and regulations. ${ }^{9,10}$ The Bioethics Commission at the Medical University of Warsaw was consulted and confirmed that our study did not require formal approval or consent. 
Table 1

Demographic data and intracranial aneurysm location

\begin{tabular}{|c|c|c|c|c|}
\hline Aneurysm & Age & Gender & Cause of death & Aneurysm location \\
\hline+ & 60 & $M$ & acute myocardial infarction & R MCA bif \\
\hline+ & 55 & $\mathrm{~F}$ & pneumonia & ACommA \\
\hline+ & 60 & M & suicidal hanging & L MCA bif \\
\hline+ & 63 & $\mathrm{~F}$ & generalized cancer & L ICA bif \\
\hline+ & 67 & $M$ & suicidal hanging & L MCA bif \\
\hline+ & 58 & $M$ & drowning & L ICA bif \\
\hline+ & 69 & $M$ & alcohol poisoning & ACommA \\
\hline+ & 61 & $M$ & alcohol poisoning & L ICA bif \\
\hline- & 56 & M & suicidal hanging & \\
\hline- & 59 & M & myocarditis & \\
\hline- & 55 & $M$ & acute myocardial infarction & \\
\hline- & 65 & $\mathrm{~F}$ & pulmonary embolism & \\
\hline- & 72 & $\mathrm{~F}$ & acute myocardial infarction & \\
\hline- & 70 & $M$ & fall from height & \\
\hline- & 52 & M & generalized cancer & \\
\hline- & 66 & M & acute myocardial infarction & \\
\hline
\end{tabular}

\section{Pressure-inflation tests}

Biomechanical experiments of sIA and CA specimens were performed in a working area, which allowed for continuous measurement of intravascular pressure with simultaneous visual registration (Figure 1A). All analyzed specimens were rinsed with $0.9 \% \mathrm{NaCl}$ to remove the blood clots. Then, a flared tip cannula was inserted into the prepared specimen. The proximal end of the specimen was ligated with a surgical suture [4.0 silk suture] to ensure tight attachment to the cannula. To form a closed system, the opposite ends, as well as minor cortical and perforating arteries, were ligated using 4.0 and 7.0 silk sutures, respectively (Figure 1B). Next, a precision dosing pump that ran $0.9 \% \mathrm{NaCl}$ at $36^{\circ} \mathrm{C}$ was activated. First, five preconditioning cycles were performed with gradually increasing-decreasing pressure ranging from 0 
to $200 \mathrm{mmHg}$ at a speed of $10 \mathrm{mmHg} / \mathrm{s}$ for muscle fiber relaxation. Then, the specimen was subjected to quasi-static increasing pressure at a rate of $76 \mathrm{mmHg} / \mathrm{s}$ until the arterial bifurcation, or the wall of the CA or sIA, ruptured (Figure 1C). The follow-up steering control system regulated the pump revolutions to provide a constant increase in pressure. Differences between the aneurysm and non-aneurysm groups in terms of rupture pressure values of specific CA segments and rupture sites of pressurized specimens were analyzed. Furthermore, association of the obtained rupture pressure values with age of the autopsy subjects was evaluated for both groups separately.

\section{Statistical analysis}

Statistical analyses were performed using the statistical package STATISTICA 13.1 (StatSoft, Inc.) and R environment. All continuous and ordinal variables were summarized as mean and standard deviation (SD). Percentages, numerators, and denominators were presented for categorical and binary variables. Student's t-test for independent samples was used to examine differences between the two groups in continuous variables. Fisher's exact test was used to examine the qualitative variables. To examine differences between more than two groups, the Kruskal-Wallis ANOVA test was used. Regardless of the results of the main analysis, post-hoc analysis was conducted using the Dunn test with Benjamini and Hochberg $p$-value adjustment to control for the false discovery rate (FDR); therefore, $p$-values corrected for multiple comparisons ( $p F D R$-corrected) are reported. Pearson's correlation was used to measure the linear relationship between variables. For all calculations, the statistical significance level was set at $a=$ 0.05 .

\section{Results}

\section{CAs rupture pressure}

The mean rupture pressure of CAs in the control group was $1048 \pm 323 \mathrm{mmHg}$, and the mean rupture pressure of CAs harvested from brains with single unruptured sIA was $1054 \pm 289 \mathrm{mmHg}$. No significant differences were observed in rupture pressure values between groups $(p=0.54)$ (Figure 2A). The average rupture pressures of ACommA were $723 \pm 199 \mathrm{mmHg}$ and $661 \pm 60 \mathrm{mmHg} ; \mathrm{BA}, 900 \pm 119 \mathrm{mmHg}$ and $971 \pm 211 \mathrm{mmHg}$; ICA, $1272 \pm 267 \mathrm{mmHg}$ and $1162 \pm 252 \mathrm{mmHg} ; \mathrm{MCA}, 1132 \pm 304 \mathrm{mmHg}$ and $1120 \pm 375$ $\mathrm{mmHg}$ in the aneurysm and non-aneurysm groups, respectively. There were no significant differences in rupture pressure of ACommA ( $p=0.42), B A(p=0.42), I C A(p=0.25)$, and MCA $(p=0.92)$ between the experimental and control groups (Table 2). Furthermore, there were significant differences in the mean rupture pressure values between specific CA segments in the aneurysm group $(p=0.0003)$. In the post-hoc analysis in the aneurysm group, the rupture pressure values of the ICA were significantly higher than those of the ACommA ( $p=0.001)$ and BA ( $p=0.01)$, as well as MCA compared to ACommA ( $p=0.01)$ (Figure $2 B$ ). Similarly, there were significant differences in the average rupture pressure values between the aforementioned CA segments in the non-aneurysm group $(p=0.0003)$. Post-hoc analysis revealed significantly lower rupture pressure values of ACommA compared to the remaining CA segments: $B A$ $(p=0.03), \operatorname{ICA}(p=0.0002)$, and MCA ( $p=0.001$, Figure $2 C)$. 
Table 2

Mean rupture pressure of specific CAs segments depending on the presence of unruptured sIA.

\begin{tabular}{|llllll|}
\hline & \multicolumn{4}{l}{ Aneurysm group } & \multicolumn{2}{l|}{ Non-aneurysm group } \\
& \multicolumn{4}{l}{ Rupture pressure } & \multicolumn{2}{l|}{ Rupture pressure } \\
& mean & SD & mean & SD & p-value \\
\hline ACommA (mmHg) & 723 & 199 & 661 & 60 & 0.42 \\
\hline BA (mmHg) & 900 & 119 & 971 & 211 & 0.42 \\
\hline ICA (mmHg) & 1272 & 267 & 1162 & 252 & 0.25 \\
\hline MCA (mmHg) & 1132 & 304 & 1120 & 375 & 0.92 \\
\hline Total (mmHg) & 1074 & 315 & 1033 & 324 & 0.54 \\
\hline $\begin{array}{l}\text { Abbreviations: ACommA, anterior communicating artery; BA, basilar artery; ICA, internal carotid artery; } \\
\text { MCA, middle cerebral artery; SD, standard deviation. }\end{array}$ & \multicolumn{5}{l}{} \\
\hline
\end{tabular}

\section{SIAs rupture pressure}

Among the eight analyzed sIAs, two were located at the ACommA complex, three at the bifurcation of the ICA, and three at the bifurcation of the MCA. Rupture of the sIA wall was identified in three specimens: ACommA, ICA, and MCA aneurysm. The average sIA rupture pressure was $769 \pm 230 \mathrm{mmHg}$. In the other five specimens, perforation occurred within the arterial wall.

Rupture site - bifurcation vs. wall of CA specimens

During the pressure-inflation tests, rupture predominantly occurred at the CA wall in $32 / 38(84 \%)$ and in $34 / 40$ (85\%) of the analyzed specimens in the experimental and control groups, respectively. Specifically, in the aneurysm group, the bifurcation region ruptured in 6/15 (40\%) of MCAs, while no rupture was observed in ICAs; meanwhile, in the non-aneurysm group, rupture was observed in 4/16 (25\%) of MCAs and $2 / 16(12.5 \%)$ of ICAs. No rupture was identified in the bifurcation region of the BA in either group. No significant differences were noted between groups regarding the rupture site of $B A(p=1), \operatorname{ICA}(p=0.48)$, and MCA $(p=0.46)$ (Table 3). Moreover, there were no significant differences regarding rupture pressure values of the MCA ( $p=0.89)$ depending on the rupture site in the group with sIA. Similarly, no significant differences were observed between the rupture pressure values of the ICA $(p=0.93)$ and MCA $(p=0.79)$ depending on the rupture site of the analyzed specimen in the control group. 
Table 3

Rupture site of specific CAs segments depending on the presence of unruptured sIA.

\begin{tabular}{|llllll|}
\hline \multicolumn{5}{|l}{ Aneurysm group } & \multicolumn{3}{l|}{ Non-aneurysm group } \\
\hline & Rupture site & \multicolumn{5}{l|}{ Rupture site } \\
\hline & Bif & Wall & Bif & Wall & p-value \\
\hline BA & 0 & $100 \%$ & 0 & $100 \%$ & 1 \\
\hline ICA & 0 & $100 \%$ & $12,5 \%$ & $87,5 \%$ & 0.48 \\
\hline MCA & $40 \%$ & $60 \%$ & $25 \%$ & $75 \%$ & 0.46 \\
\hline Total & $16 \%$ & $84 \%$ & $15 \%$ & $85 \%$ & 1 \\
\hline $\begin{array}{l}\text { Abbreviations: ACommA, anterior communicating artery; BA, basilar artery; ICA, internal carotid artery; } \\
\text { MCA, middle cerebral artery; Bif, bifurcation. }\end{array}$ \\
\hline
\end{tabular}

\section{Age dependency of rupture pressure values}

No significant differences were noted between the mean age of autopsy subjects in the aneurysm and non-aneurysm groups $(p=0.83)$. The mean CA rupture pressure in the aneurysm group was negatively correlated with age $(r=-0.45, p=0.002)$. In contrast, there was no significant correlation between age and mean $C A$ rupture pressure in the non-aneurysm group $(p=0.508)$ (Figure $3 A-B)$.

\section{Discussion}

\section{Loci minoris resistentiae of the cerebral arterial network}

In our study, the presence of single unruptured sIA did not influence the rupture pressure values of specific CA segments. Moreover, bifurcation regions displayed high strength, and the rupture pressure did not significantly differ based on location of the rupture of the analyzed CAs, specifically at the wall or bifurcation region. Nevertheless, based on the study of Mitchell and Jakubowski, the average rupture pressure of branched CA specimens was significantly lower than the rupture pressure of straight CA specimens. ${ }^{11}$ Alternatively, Ciszek et al. did not observe a significant difference in rupture pressure values between straight and branched CA specimens. ${ }^{12}$ In our study, rupture occurred most frequently at the wall of the analyzed CAs. During the pressure-inflation tests, the bifurcation region ruptured in $40 \%$ of MCAs from the aneurysm group and in $25 \%$ of MCAs and $12.5 \%$ of ICAs from the non-aneurysm group. However, such high strength of CA bifurcations may be counter-intuitive when considering that bifurcation regions are histologically characterized by a discontinuity of the media ${ }^{13}$ and enlarged fenestrations within the IEL. ${ }^{3}$ Finlay et al. indicated that the arrangement of adventitial collagen fibers at 
the bifurcation apex differs from that in the straight arterial segments. In the bifurcation region, collagen fibers are densely co-aligned and form a strong, tendon-like band that provides high strength of the CA bifurcation. ${ }^{14}$

The sIA development comprises a sequence of overlapping adaptive and degenerative processes induced by specific hemodynamic factors. Only when degenerative processes prevail over adaptive processes does wall strength decrease, leading to sIA rupture. ${ }^{8}$ Thus, not all sIAs correspond to regions of low wall strength. In a study by Ciszek et al., one sIA ruptured under pressure markedly exceeding physiological blood pressure, while another sIA sustained high intraluminal pressure values. ${ }^{12}$ Additionally, during the experiments conducted by Mitchell and Jakubowski, the sIA specimen was pressurized up to $1.6 \mathrm{bar}$ $(1200 \mathrm{mmHg})$ and the rupture occurred within the wall of adjacent CA. ${ }^{11}$ Similarly, in our study, none of the analyzed sIAs ruptured at physiological pressures. Furthermore, in the case of five sIA specimens, the rupture occurred remotely from the sIA, indicating its high wall strength. According to the lifelong Finnish cohort follow-up study, approximately $70 \%$ of slAs did not rupture during the lifetime observation ${ }^{15}$ which is in line with the results of our study, as well as the aforementioned CAs pressure-inflation test studies.

Specific CA segments that are prone to sIA formation simultaneously predispose to an increased risk of sIA rupture. In a retrospective epidemiological study by Carter et al., ruptured sIAs were most frequently located in the ACommA complex. Additionally, the mean size of sIAs involving ACommA or distal branches of major CAs was smaller than that of sIAs observed at larger CAs, such as the ICA or MCA bifurcation. Carter et al. hypothesized that there is a decrease in sIA wall thickness along with a decrease in the caliber of the parent artery. ${ }^{16}$ It may be reasonable to consider Laplace's law applied to this context: when assuming similar pressure conditions and the same diameter, sIAs with thin walls experience remarkably higher intramural stress than their thick-wall counterparts. Likewise, in our study, ACommA displayed significantly lower mean rupture pressure than the ICA and MCA bifurcation in both the aneurysm and non-aneurysm groups. However, in a study by Ciszek et al., as well as in a study by Mitchell and Jakubowski, the authors did not observe significant differences between rupture pressure values depending on the CA segment location. ${ }^{11,12}$ Further investigations, including histological studies with the measurement of wall thickness, are required to clarify whether caliber of CAs is related to the strength of their wall.

\section{Mechanobiology of the CAs}

The rupture pressure values of CAs in both analyzed groups markedly exceeded the maximal in vivo arterial blood pressure ${ }^{17}$ which is consistent with the results of earlier pressure-inflation tests of CAs. In a study of Mitchell and Jakubowski, the average rupture pressure of all analyzed CAs was 1.848 bar (1386 $\mathrm{mmHg}$ ), while the mean CAs rupture pressure presented in a study conducted by Ciszek et al. was 2.35 atm $(1786 \mathrm{mmHg}) .{ }^{11,12}$ Such discrepancies in the literature data regarding rupture pressure values may result from the diverse age distribution of CAs donors. The mean age at the time of death of autopsy subjects was 69 years in the former study and 47 years in the latter study. Additionally, both studies 
revealed a significant negative dependency of CA rupture pressure with age. Thus, the relatively higher mean CA rupture pressure obtained by Ciszek et al. may be explained by the predominance of CAs harvested from younger autopsy subjects.

Gradual decrease of the rupture pressure of CAs with age, similar to the gradual increase with age of CA stiffness, may be attributable to age-related arterial wall remodeling, including IEL degeneration. ${ }^{4}$ Specifically, elastin is the main load-bearing material at low strain conditions, while collagen fibers maintain structural integrity of the CA during further increase of mechanical loadings. Progressive elastin degradation results in an age-related increase in CA stiffness at low strain regimes, indicating early recruitment of collagen fibers. Since collagen fibers are capable of slight deformations prior to failure, once the elastin function is impaired, the intramural stress within the CA wall increases under the same pressure conditions. ${ }^{18}$ Therefore, it may be conjectured that a significant decrease in rupture pressure values with age exclusively among CAs in the aneurysm group indicates more pronounced progression of elastin degradation within the arterial wall compared to CAs in the non-aneurysm group. However, we did not find a significant difference between the experimental and control groups in terms of mean CA rupture pressure. This discrepancy may be explained by the narrow range of age distribution of CAs donors. If CAs from younger and older autopsy subjects had been pressurized, then CAs from the nonaneurysm group may have presented similar negative dependency of rupture pressure with age.

\section{Limitations}

The comparison of rupture pressure values between sIAs and corresponding CA segments was limited by the small number of ruptured sIAs during the pressure-inflation tests. Also, all the analyzed CA and sIA specimens were obtained from the autopsy subjects and the experiments were conducted within 36 hours postmortem. Furthermore, in the case of CAs harvested from human autopsy subjects, the smooth muscles of the wall are consequently without tone. Due to differences between biomechanical properties of CAs collected during surgery and autopsy, presented results may not completely reflect in vivo wall strength of both CAs and sIAs. ${ }^{19}$ Alternatively, only in the case of pressure-inflation tests conducted on the specimens harvested from the autopsy subjects, rupture pressure values of sIAs and major CAs may be assessed.

\section{Conclusions}

The mean CA rupture pressure did not significantly differ between the aneurysm and non-aneurysm groups, and markedly exceeded the maximal in vivo arterial blood pressure values. Thus, it may be concluded that the presence of unruptured sIA is not related to the generalized weakening of the CAs. Moreover, $\mathrm{CA}$ bifurcations were not identified as regions of decreased wall strength. Alternatively, location of the ACommA of the analyzed CA segment and older age of the autopsy subject are associated with lower rupture pressure values. Nevertheless, in our study, a significant negative correlation between CA 
rupture pressure values and age associated with sIA presence requires further research conducted on CAs donors with a greater age distribution.

\section{Declarations}

\section{Data availability:}

The data that support the findings of this study are available from the corresponding author upon reasonable request.

\section{Acknowledgments:}

We would like to thank Editage (www.editage.com) for English language editing.

\section{Author contribution statement:}

LL - manuscript writing, acquisition of data, study concept and design, analysis and interpre-tation of data; PK - study concept and design, analysis and interpretation of data, study supervision; ST acquisition of data, study concept and design; AP - acquisition of data, analysis and interpretation of data; SK - statistical analysis of data; AM - study concept and design, critical revision of manuscript for intellectual content

All authors reviewed and approved the final manuscript.

\section{Additional information:}

The authors declare no competing interests.

\section{References}

1. Rinkel, G.J., Djibuti, M., Algra, A. \& van Gijn, J. Prevalence and risk of rupture of intracranial aneurysms: a systematic review. Stroke 29, 251-256 (1998).

2. Rinkel, G.J. Medical management of patients with aneurysmal subarachnoid haemorrhage. Int $J$ Stroke 3, 193-204 (2008).

3. Campbell, G.J. \& Roach, M.R. Fenestrations in the internal elastic lamina at bifurcations of human cerebral arteries. Stroke 12, 489-496 (1981).

4. Fonck, E., et al. Effect of aging on elastin functionality in human cerebral arteries. Stroke 40, 25522556 (2009).

5. Kaspera, W., et al. Morphological and Hemodynamic Risk Factors for Middle Cerebral Artery Aneurysm: a Case-Control Study of 190 Patients. Sci Rep 10, 2016 (2020).

6. Zhang, X.J., Hao, W.L., Zhang, D.H. \& Gao, B.L. Asymmetrical middle cerebral artery bifurcations are more vulnerable to aneurysm formation. Sci Rep 9, 15255 (2019). 
7. Toth, M., Nadasy, G.L., Nyar, I., Kerenyi, T. \& Monos, E. Are there systemic changes in the arterial biomechanics of intracranial aneurysm patients? Pflugers Arch 439, 573-578 (2000).

8. Frosen, J., et al. Saccular intracranial aneurysm: pathology and mechanisms. Acta Neuropathol 123, 773-786 (2012).

9. Ustawa z dnia 5 grudnia 1996 r. o zawodach lekarza i lekarza dentysty (Dz. U. 1997, vol. 28, item 152, with amendments) [Act on Medical Doctors and Dentists], Article 21(1).

http://isap.sejm.gov.pl/isap.nsf/download.xsp/WDU19970280152/U/D19970152Lj.pdf

10. Ustawa z dnia 1 lipca 2005 r. o pobieraniu, przechowywaniu i przeszczepianiu komórek, tkanek i narządów (Dz. U. 2005, vol. 169, item 1411, with amendments) [Act on the collection, storage and transplantation of cells, tissues and organs], Article 4(1) and 4(2). http://isap.sejm.gov.pl/isap.nsf/download.xsp/WDU20051691411/U/D20051411Lj.pdf

11. Mitchell, P. \& Jakubowski, J. Failure testing cerebral arteries: are branch points weaker than unbranched vessels? Br J Neurosurg 16, 578-582 (2002).

12. Ciszek, B., Cieslicki, K., Krajewski, P. \& Piechnik, S.K. Critical pressure for arterial wall rupture in major human cerebral arteries. Stroke 44, 3226-3228 (2013).

13. Stehbens, W.E. Medial defects of the cerebral arteries of man. J Pathol Bacteriol 78, 179-185 (1959).

14. Finlay, H.M., Whittaker, P. \& Canham, P.B. Collagen organization in the branching region of human brain arteries. Stroke 29, 1595-1601 (1998).

15. Korja, M., Lehto, H. \& Juvela, S. Lifelong rupture risk of intracranial aneurysms depends on risk factors: a prospective Finnish cohort study. Stroke 45, 1958-1963 (2014).

16. Carter, B.S., Sheth, S., Chang, E., Sethl, M. \& Ogilvy, C.S. Epidemiology of the size distribution of intracranial bifurcation aneurysms: smaller size of distal aneurysms and increasing size of unruptured aneurysms with age. Neurosurgery 58, 217-223; discussion 217-223 (2006).

17. MacDougall, J.D., Tuxen, D., Sale, D.G., Moroz, J.R. \& Sutton, J.R. Arterial blood pressure response to heavy resistance exercise. J Appl Physiol (1985) 58, 785-790 (1985).

18. Scott, S., Ferguson, G.G. \& Roach, M.R. Comparison of the elastic properties of human intracranial arteries and aneurysms. Can J Physiol Pharmacol 50, 328-332 (1972).

19. Monson, K.L., Goldsmith, W., Barbaro, N.M. \& Manley, G.T. Significance of source and size in the mechanical response of human cerebral blood vessels. J Biomech 38, 737-744 (2005).

\section{Figures}

\section{Figure 1}

A. A block diagram of a working area. Following the activation (1), temperature controller regulates the temperature of the $0.9 \% \mathrm{NaCl}(2)$ maintaining its predefined value and sends a set point to pressure 
regulator (3). The feedback signal from the pressure transducer (4) regulator maintains proper pressure within analysed CA or sIA specimen by controlling the precision dosing pump (5). Multicolor lights (6) provide optimal conditions for visual registration. LED diodes (7) were used to correlate the pressure with the image from the camera (8). B. BA bifurcation specimen mounted on a flared tip cannula before the pressurization. Both opposite ends, i.e., posterior cerebral arteries, as well as perforating branches, are ligated with 4.0 and 7.0 silk sutures, respectively. C. ICA bifurcation specimen at the time of rupture within the bifurcation region during pressure-inflation test; black arrow indicates the stream of $0.9 \% \mathrm{NaCl}$.

\section{Figure 2}

A. Comparison of rupture pressure values of CAs between the aneurysm and non-aneurysm groups. B. Comparison of rupture pressure values of specific CA segments in the aneurysm and C. non-aneurysm group. Data represent violin plots, red dots indicate the arithmetic mean value, a horizontal black line inside the frame represents the median value, dots inside the graphs indicate the results of individual patients (dot positions along the abscissa are set to increase their visibility), and the shape of the graph indicates the distribution of values.

\section{Figure 3}

A. Relationship between rupture pressure values of CAs and age of the autopsy subjects in the aneurysm and B. non-aneurysm group. Graphs display median (straight line) and 95\% confidence intervals (curves). 